\title{
Regulation of IncRNA-H19/miR-140-5p in cartilage matrix degradation and calcification in osteoarthritis
}

\author{
Bo Yang, Lei Xu, Shuang Wang \\ Department of Nephrology Endocrinology and Rheumatology, The 960th Hospital of the Chinese People's Liberation Army, Zibo 255300, China \\ Contributions: (I) Conception and design: B Yang, S Wang; (II) Administrative support: L Xu; (III) Provision of study materials or patients: All \\ authors; (IV) Collection and assembly of data: All authors; (V) Data analysis and interpretation: B Yang, S Wang (VI) Manuscript writing: All authors; \\ (VII) Final approval of manuscript: All authors \\ Correspondence to: Shuang Wang. The 960th Hospital of the Chinese People's Liberation Army Department of Nephrology Endocrinology and \\ Rheumatology, 20 Zhanbei Road, Zhoucun District, Zibo City, Zibo 255300, China. Email: wangshuangsw@sina.com.
}

Background: To study the effect of long non-coding RNA H19/microRNA (miR)-140-5p regulatory axis on the degradation and calcification of osteoarthritis (OA) cartilage matrix.

Methods: Twenty cases of clinical OA cartilage tissue and healthy cartilage tissue were collected. Construction of small interfering RNA vector (si-H19) and negative control (si-Control), miR-140-5p mimic and negative control mimic-NC, miR-140-5p inhibitor, and negative control inhibitor-NC transfected human chondrocytes HC-A cells. Chondrocytes were categorized into classes of si-H19, si-Control, mimic, mimic-NC, inhibitor, NC inhibitor, inhibitor + si-H19, NC inhibitor + si-H19. The expression levels of H19, miR-140-5p, alkaline phosphatase (ALP), osteocalcin (OCN), and bone sialoprotein (BSP) in cartilage tissue and chondrocytes were detected using real-time fluorescent quantitative PCR (RT-qPCR). In each group of chondrocytes, Western blotting was used to detect the levels of matrix metalloproteinase (MMP)1, MMP-13, and type II $\alpha 1$ collagen fibers (COL2A1). CCK- 8 was used to detect the cell proliferation rate, and flow cytometry was used to detect the cell apoptosis rate.

Results: H19 was up-regulated in OA cartilage tissue $(\mathrm{P}<0.05)$; Silencing $\mathrm{H} 19$ not only prevented chondrocyte apoptosis but also facilitated chondrocyte proliferation (both $\mathrm{P}<0.05$ ), down-regulated MMP1 and MMP-13 expression levels (both $\mathrm{P}<0.05)$, and up-regulated COL2A1 expression rates $(\mathrm{P}<0.05)$. Also, silenced H19 inhibited the mRNA level of ALP, OCN, and BSP and ALP activity of chondrocytes (all $\mathrm{P}<0.05$ ). There was a negative correlation between miR-140-5p and H19 in OA cartilage tissue and chondrocytes $(r=-0.98, \mathrm{P}<0.001)$. Silenced miR-140-5p significantly reversed the inhibitory effects of silenced H19 on matrix degradation and calcification markers in chondrocytes (all $\mathrm{P}<0.05)$.

Conclusions: The present study showed that the H19/ miR-140-5p regulatory axis could affect the degradation and calcification of the cartilage matrix in OA cells.

Keywords: Long non-coding RNA H19 (lncRNA H19); miR-140-5p; osteoarthritis (OA); cartilage; mechanism degradation; calcification

Submitted Mar 19, 2020. Accepted for publication May 26, 2020.

doi: 10.21037/apm-20-929

View this article at: http://dx.doi.org/10.21037/apm-20-929

\section{Introduction}

Osteoarthritis (OA) is a chronic degenerative joint disease, whose incidence may increase with age and more common in the elderly. OA is defined by insufficient cartilage matrix synthesis, degeneration of the articular cartilage, development of an osteophyte, and synovitis. The pathogenesis of OA involves various physiological and pathological processes of the body, including articular cartilage anabolic dysfunction, catabolism, and degradation 
of the extracellular matrix (1). However, the exact mechanism of $\mathrm{OA}$ is unclear.

In OA, the epigenetic modifications of long non-coding RNA (lncRNA) and microRNA (miRNA) have been reported to play a vital part $(2,3)$. For example, HOTTIP expression is up-regulated, while homeobox gene A13 expression is down-regulated in OA cartilage tissue, which inhibits integrin $\alpha 1$ synthesis and causes cartilage destruction (4). Down-regulation of lncRNA MEG3 in OA cartilage tissue leads to OA progression via regulating the miR-16/SMAD7 axis (5). Like lncRNA, expression changes in various miRNAs can also affect OA. For example, the up-regulation of miR-146a and miR-98 can promote the progress of OA (3), and miR-16-5p also participates in the occurrence and development of OA (5). In addition, exosomes carrying miR-140-5p are reported to promote the proliferation and migration of rat chondrocytes in $\mathrm{OA}$ without disrupting the secretion of extracellular matrixes (6).

Studies have shown that the expression of IncRNA H19 increased in OA cartilage tissue to regulate miR675 and affect synthesis and catabolism of cartilage, which speculates that H19 is a potential target for stimulating cartilage recovery (7). However, the effects of expression changes of H19 on the degradation and calcification of the OA cartilage matrix are unclear. In this report, it was indicated that H19 could target miR-140-5p to inhibit its expression, and the regulatory axis H19/miR-140-5p had a potential role in regulating degradation and calcification of the matrix of the OA cartilage. This study may supply a potential target and basis for the diagnosis and treatment of OA chondrocyte degeneration.

\section{Methods}

\section{Clinical data}

Cartilage tissue samples from 20 patients with primary knee $\mathrm{OA}$ who underwent joint replacement. Samples of knee cartilage tissue from 20 post-traumatic amputation patients were obtained in our hospital's orthopedic department from January 2015 to June 2019. The trial was conducted in accordance with the Declaration of Helsinki (as revised in 2013). All specimens were approved by our ethics committee and get the informed consent of the patients. Among the twenty patients with primary knee OA, 13 males and seven females were included, with an average age of 52.6 years old (ranging from 39 to 72 years). There were 18 cases of traumatic amputation, including 12 males and six females, with an average age of 41.8 years old (ranging from 29 to 61 years).

\section{Reagents and consumables}

TRIzol kit was bought from Invitrogen (USA). PrimeScript TM RT Master Mix and LipofecamineTM2000 kits were obtained from Eurofins MWG Operon (Germany). Human chondrocyte cell lines (HC-A) were purchased from Shanghai Aolu Biotechnology Co., Ltd. SYBR qPCR Mix and cell counting kit 8 (CCK-8) was purchased from TaKaRa (Japan), and the phosphatase detection kit was purchased from China Jiancheng Biological Engineering Company. The bicinchoninic acid (BCA) kit was bought from Thermo Fisher Scientific (USA). Polyvinylidene fluoride (PVDF) membrane was bought from Millipore (USA). The primary antibodies against MMP-1, MMP13, type II collagen $\alpha 1$ (COL2A1), GAPDH, and relative secondary antibodies were all bought from Abcam (USA).

\section{Total RNA extraction and real-time quantitative polymerase chain reaction ( $R T-q P C R)$}

The TRIzol kit was used to extract complete RNA from cartilage tissue, relative healthy tissues, and chondrocytes, and a UV spectrophotometer was used to measure RNA concentrations. Complementary DNA (cDNA) was synthesized according to the instructions of the PrimeScript TM RT Master Mix kit, under the following reaction conditions: $40{ }^{\circ} \mathrm{C}$ for $6 \mathrm{~min}, 65^{\circ} \mathrm{C}$ for $25 \mathrm{~min}$. Finally, we got the cDNA product to store at $-80{ }^{\circ} \mathrm{C}$ for a further experiment in $\mathrm{qPCR}$. The reaction system of RT-qPCR was at a total volume of $20 \mu \mathrm{L}$, including $2 \mu \mathrm{L}$ cDNA product, $0.4 \mu \mathrm{L} 50 \times$ ROX, $10 \mu \mathrm{L}$ SYBR qPCR Mix, $0.8 \mu \mathrm{L}$ upstream primer and $0.8 \mu \mathrm{L}$ downstream primer and RNase water supplemented to $20 \mu \mathrm{L}$. The reaction conditions were as follows that first denaturation at $95^{\circ} \mathrm{C}$ for $1 \mathrm{~min}$, then $95^{\circ} \mathrm{C}$ for $30 \mathrm{~s}$ and $60^{\circ} \mathrm{C}$ for $40 \mathrm{~s}$, for a total of 40 cycles. Each experiment was performed in 3 replicates, and each sample was repeated for three times. The relative expression of a gene was expressed using $2^{-\Delta \Delta \mathrm{Ct}}$ using a relative quantitative method. All steps for this experiment were performed on ice. Primer sequences were shown in Table 1.

\section{Cell culture}

HC-A cells were cultured in Dulbecco's Modified Eagle's Medium (DMEM)/F12 medium, having 10\% fetal bovine 
Table 1 Primer sequences for PCR

\begin{tabular}{ll}
\hline Genes & Primer sequences \\
\hline H19 & Forward: 5'-GCAAGAAGCGGGTCTGTTT-3' \\
& Reverse: 5'-GCTGGGTAGCACCATTTCTT-3' \\
miR-140-5p & Forward: 5'-TGCGGCAGTGGTTTACCCTATG-3' \\
& Reverse: 5'-CCAGTGCAGGGTCCGAGGT-3' \\
U6 & Forward: 5'-TGCGGGTGCTCGCTTCGGCAGC-3' \\
& Reverse: 5'-CCAGTGCAGGGTCCGAGGT-3' \\
ALP & Forward: 5'-CCAACTCTTTTGTGCCAGAGA-3' \\
& Reverse: 5'-GGCTACATTGGTGTTGAGCTTT-3' \\
OCN & Forward: 5'-AGCAAAGGTGCAGCCTTTGT-3' \\
& Reverse: 5'-GCGCCTGGGTCTCTTCACT-3' \\
BSP & Forward: 5'-TGCTCAGCATTTGGGAAT-3' \\
& Reverse: 5'-TGCATTGGCTCCAGTGACACT-3' \\
GAPDH & Forward: 5'-ATGGTGAAGGTCGGTGTGAA-3' \\
& Reverse: 5'-GAGTGGAGTCATACTGGAAC-3' \\
\hline
\end{tabular}

serum. All the cells were cultured at $37{ }^{\circ} \mathrm{C}$ in a humid sterile incubator holding $5 \% \mathrm{CO}_{2}$, with the medium changed once every two days.

\section{Cell transfection}

The cells were seeded into plates with 6 wells. After 24 h, when cell density reached $70 \%$, cells were divided into six treatment groups including H19 interference group (siH19), negative transfection group (si-Control), miR-140$5 \mathrm{p}$ mimic group (mimic), mimic negative control group (mimic-NC) and miR-140-5p inhibitor group (inhibitor) and negative control group (inhibitor-NC) inhibitor group. For transfection, mix LipofecamineTM2000 with si-H19, si-Control, mimic, mimic-NC, inhibitor, inhibitor-NC, inhibitor + si-H19, inhibitor-NC + si-H19 separately to make the final concentration of $100 \mathrm{nmol} / \mathrm{L}$ firstly, add the mixture to the cells and incubate for $6 \mathrm{~h}$, then change to the complete medium and culture for $24 \mathrm{~h}$. Finally, cells were collected for later experiments.

\section{Cell proliferation assay}

After transfection of si-H19 or si-Control, cells were collected and seeded into 96-well plates at a density of
1,000 cells/well, which were cultured for $0,24,48$, or $72 \mathrm{~h}$. The CCK-8 kit solution was added to each well at a final concentration of $10 \%$ and incubated for $1 \mathrm{~h}$ in the dark. Finally. The absorbance (OD) values were detected using a microplate reader at the wavelength of $450 \mathrm{~nm}$.

\section{Analysis of apoptosis by flow cytometry}

After transfection with si-H19 and si-Control, cells in the logarithmic growth phase were collected and digested, which were next washed twice with cold PBS and resuspend using $1 \mathrm{~mL}$ of the combined buffer to make the cell density of $1 \times 10^{6} / \mathrm{mL} .100 \mu \mathrm{L}$ of cell suspension was added to a $5 \mathrm{~mL}$ flow tube, followed by $10 \mu \mathrm{L}$ Annexin-V-FITC and $10 \mu \mathrm{L}$ propidium iodide (PI) for staining. Subsequently, it was incubated at room temperature for 15 minutes, avoiding the light. Finally, the apoptotic rate was analyzed by flow cytometry. All determinations were performed in three independent experiments.

\section{Western blotting}

The total protein of the transfected cells was extracted, then the protein concentration was determined by the BCA kit (Thermo Fisher Scientific, Waltham, Massachusetts, USA). After denatured, $20 \mu \mathrm{g}$ of total protein was separated by running sodium lauryl sulfate-polyacrylamide gel electrophoresis (SDS-PAGE) and transferred to a polyvinylidene fluoride (PVDF) membrane (Millipore, Billerica, MA, United States). After being blocked in 5\% skim milk, the membrane was incubated with primary antibodies against MMP-1, MMP-13, COL2A1, and GAPDH overnight at $4{ }^{\circ} \mathrm{C}$. Then the membrane was washed with Tris-buffered saline containing Tween 20 (TBST), which was then incubated with the secondary antibody for $2 \mathrm{~h}$ at room temperature and washed with TBST. At last, signals of protein bands could be detected after reacting with enhanced chemiluminescence (ECL) western blot detection reagents. The gray value of the bands was analyzed using ImageJ software, with GAPDH as a loading control.

\section{MMPs activity detection}

The experiments were strictly carried out according to the instructions pro-MMP-1 and MMP-13 ELISA kits (R\&D, USA). The sensitivity of the kit was 0.02 and $0.009 \mathrm{ng} / \mathrm{mL}$, respectively. 

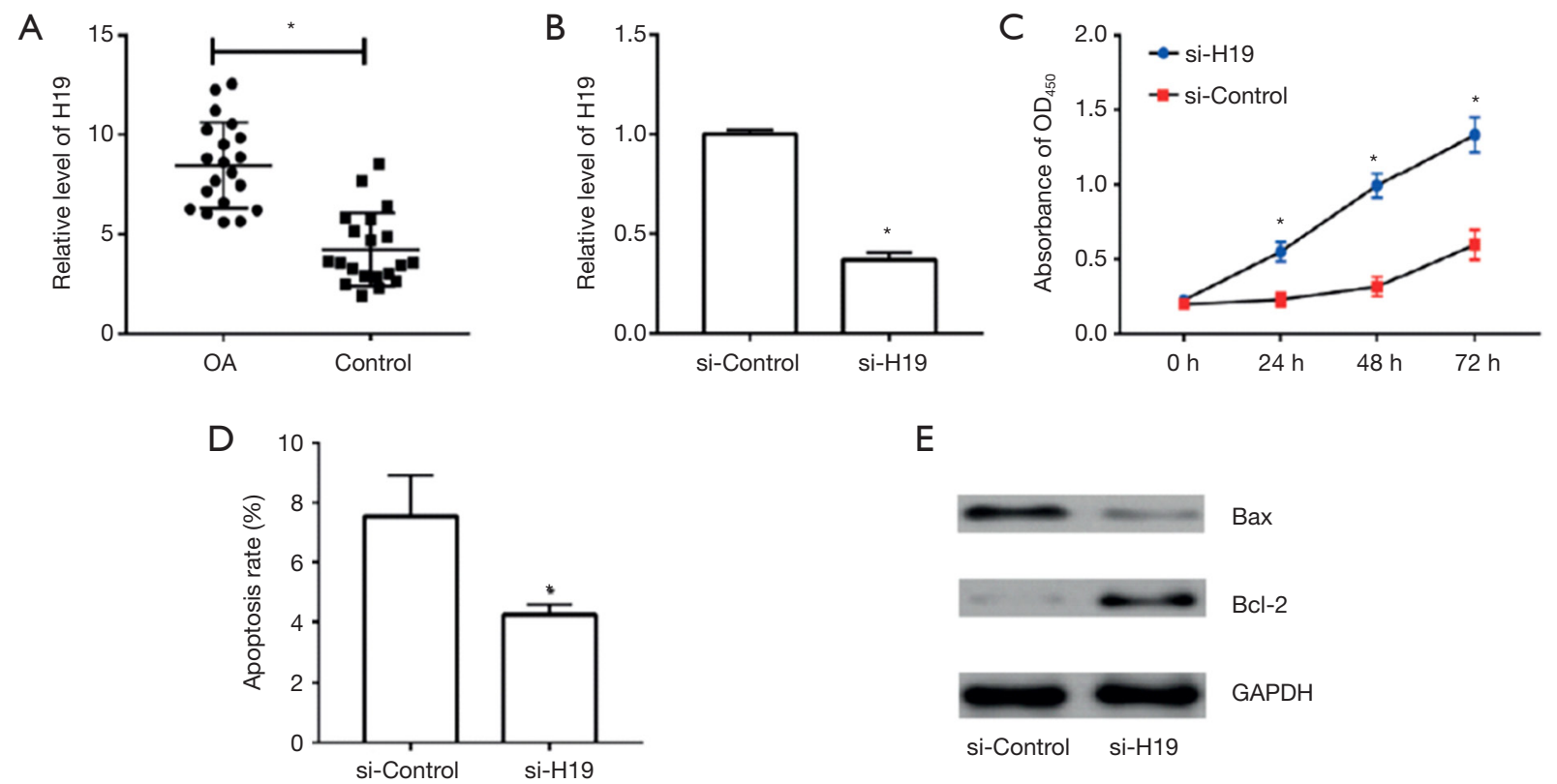

Figure $1 \mathrm{H} 19$ expression in cartilage tissue and the effects of H19 silence on chondrocyte function. (A) H19 expression levels in healthy cartilage tissue and OA cartilage tissue; ${ }^{*} \mathrm{P}<0.05$. (B) Effect of si-H19 transfection on $\mathrm{H} 19$ level of chondrocytes, compared with si-Control, ${ }^{*} \mathrm{P}<0.05$. (C) CCK-8 test was performed to detect the effect of $\mathrm{H} 19$ silence on chondrocytes proliferation, compared with si-Control, ${ }^{*} \mathrm{P}<0.05$. (D) Apoptotic rate changes detected by flow cytometry, compared with si-Control, ${ }^{*} \mathrm{P}<0.05$. (E) Detection of protein levels of apoptosis indicators (Bax and Bcl-2) by Western blotting. OA, osteoarthritis; CCK-8, cell counting kit 8 .

\section{Measurement of alkaline phosphatase (ALP)}

ALP activity was detected using an ALP detection kit. Cells from each transfection group were washed with PBS and lysed with a solution containing $20 \mathrm{mM}$ Tris- $\mathrm{HCl}(\mathrm{pH}$ 8.0), $150 \mathrm{mM} \mathrm{NaCl}, 1 \%$ Triton $\mathrm{X}-100,0.02 \% \mathrm{NaN} 3$, and 1 Triton aprotinin. The lysate was homogenized using a pipette, then incubated at $37^{\circ} \mathrm{C}$ for $15 \mathrm{~min}$. The ALP activity was measured using a spectrophotometer.

\section{Statistical analysis}

All statistical analyses were performed using SPSS 17.0 software, with all data being expressed as mean \pm standard deviation. Comparisons between groups were performed by $t$-test or one-way ANOVA, with $\mathrm{P}<0.05$ considered statistically significant, where one-way ANOVA was performed for post hoc test.

\section{Results}

\section{Expression and function of $\mathrm{H} 19$ in $\mathrm{OA}$}

Compared with healthy tissues, the expression of H19 in OA cartilage tissue increased significantly $(\mathrm{P}<0.05$, Figure $1 A)$. Transient transfection of si-H19 into could inhibit the expression of $\mathrm{H} 19$ significantly $(\mathrm{P}<0.05$, Figure $1 B)$, which could promote cell proliferation significantly and maintained for at least $72 \mathrm{~h}$ due to the silence of H19 $(\mathrm{P}<0.05$, Figure 1C). The result of flow cytometry showed that silencing $\mathrm{H} 19$ by transfection of si-H19 could inhibit apoptosis significantly $(\mathrm{P}<0.05)$ (Figure 1D). Also, si-H19 in HC-A cells could lead to low expression of Bax and high expression of Bcl-2 (Figure 1E), with the difference statistically significant $(\mathrm{P}<0.05)$.

\section{Effect of H19 on extracellular matrix-related genes}

The expression of MMP-1 and MMP-13 was suppressed by transient transfection of si-H19 in HC-A cells, while the expression of COL2A1 increased, with the difference statistically significant (both $\mathrm{P}<0.05$, Figure $2 A, B$ ).

\section{Effect of H19 on related indexes of chondrocyte ossification}

After the transfection of si-H19, mRNA levels of ALP, OCN, and BSP were down-regulated significantly (all $\mathrm{P}<0.05$ ), as 
A

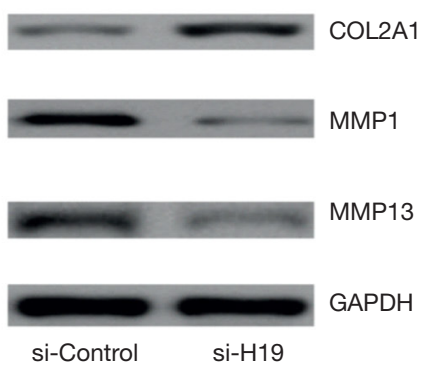

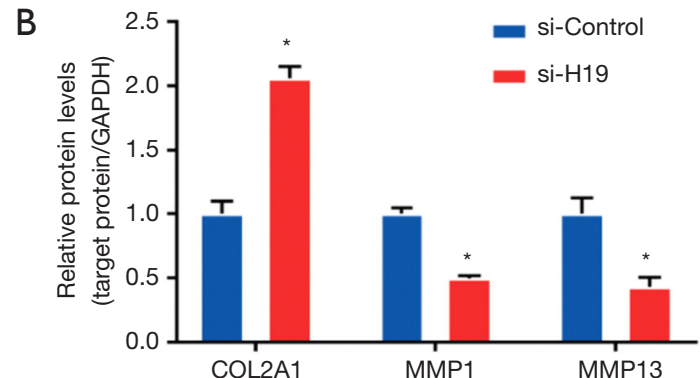

Figure 2 The effects of the si-H19 transfection in the chondrocyte on the matrix degradation-related indicators. The data compared the included MMP-1, MMP-13, and COL2A1 and the si-Control, ${ }^{*} \mathrm{P}<0.05$.
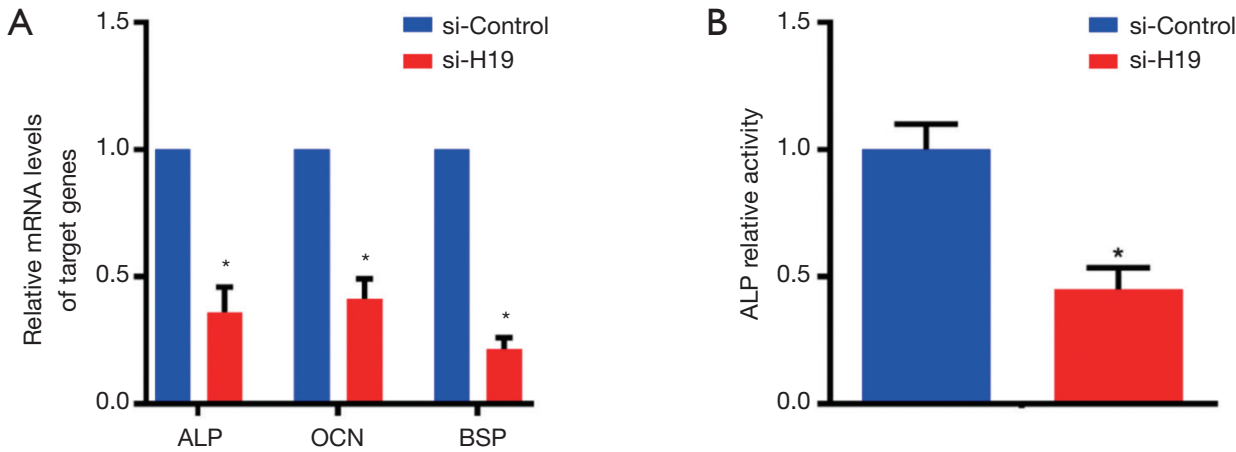

Figure 3 Effect of chondrocytes after si-H19 transfection on ossification-related indicators. (A) The levels of ALP, OCN, and BSP in the cells were detected by RT-qPCR. (B) The activity of ALP in chondrocytes was detected by the detection kit. All the results in the si-H19 transfection group were compared with si-Control, ${ }^{*} \mathrm{P}<0.05$. ALP, alkaline phosphatase; OCN, osteocalcin; BSP, bone sialoprotein.

shown in Figure 3A. Moreover, si-H19 transfection could also inhibit the ALP activity of chondrocytes, with statistical significance $(\mathrm{P}<0.05$, Figure $3 B)$.

\section{miR-140-5p is negatively regulated by $\mathrm{H} 19$}

Compared with healthy tissues, the expression of miR-140$5 \mathrm{p}$ was decreased in $\mathrm{OA}$ cartilage tissues significantly $(\mathrm{P}<0.05$, Figure $4 A$ ). It was shown that the expression of $\mathrm{H} 19$ and miR-140-5p was negatively correlated $(\mathrm{r}=-0.98, \mathrm{P}<0.001$, Figure $4 B$ ). The expression of miR-140-5p could also be upregulated by si-H19 significantly $(\mathrm{P}<0.05$, Figure $4 C)$. The expression of miR-140-5p could be increased by transfection of miR-140-5p mimic, while it could be inhibited by transfection of miR-140-5p inhibitor (Figure 4C).

\section{Effect of H19/miR-140-5p on extracellular matrix-related genes}

Chondrocytes were simultaneously transfected with miR- 140-5p inhibitor and si-H19. Compared with the si-H19 group, the expression level and activity of MMP-1 and MMP-13 were both up-regulated, while the expression level of COL2A1 was down-regulated, where both the differences were statistically significant $(\mathrm{P}<0.05$, Figure $5 A, B)$. Also, compared with the si-H19 + inhibitor-NC group, the expression level and activity of MMP-1 and MMP-13 were both up-regulated, while the expression of COL2A1 was down-regulated in the si-H19 + miR-140-5p inhibitor group, where both the differences were statistically significant $(\mathrm{P}<0.05$, Figure $5 A, B)$.

\section{Effect of H19/miR-140-5p on related indexes of chondrocyte ossification}

After transfecting with miR-140-5p inhibitor and siH19 simultaneously, the mRNA level of ALP, OCN and BSP were up-regulated compared with the si-H19 group alone (all $\mathrm{P}<0.05$, Figure $6 A$ ), while the ALP activity of chondrocytes was inhibited significantly $(\mathrm{P}<0.05$, Figure $6 B)$. 

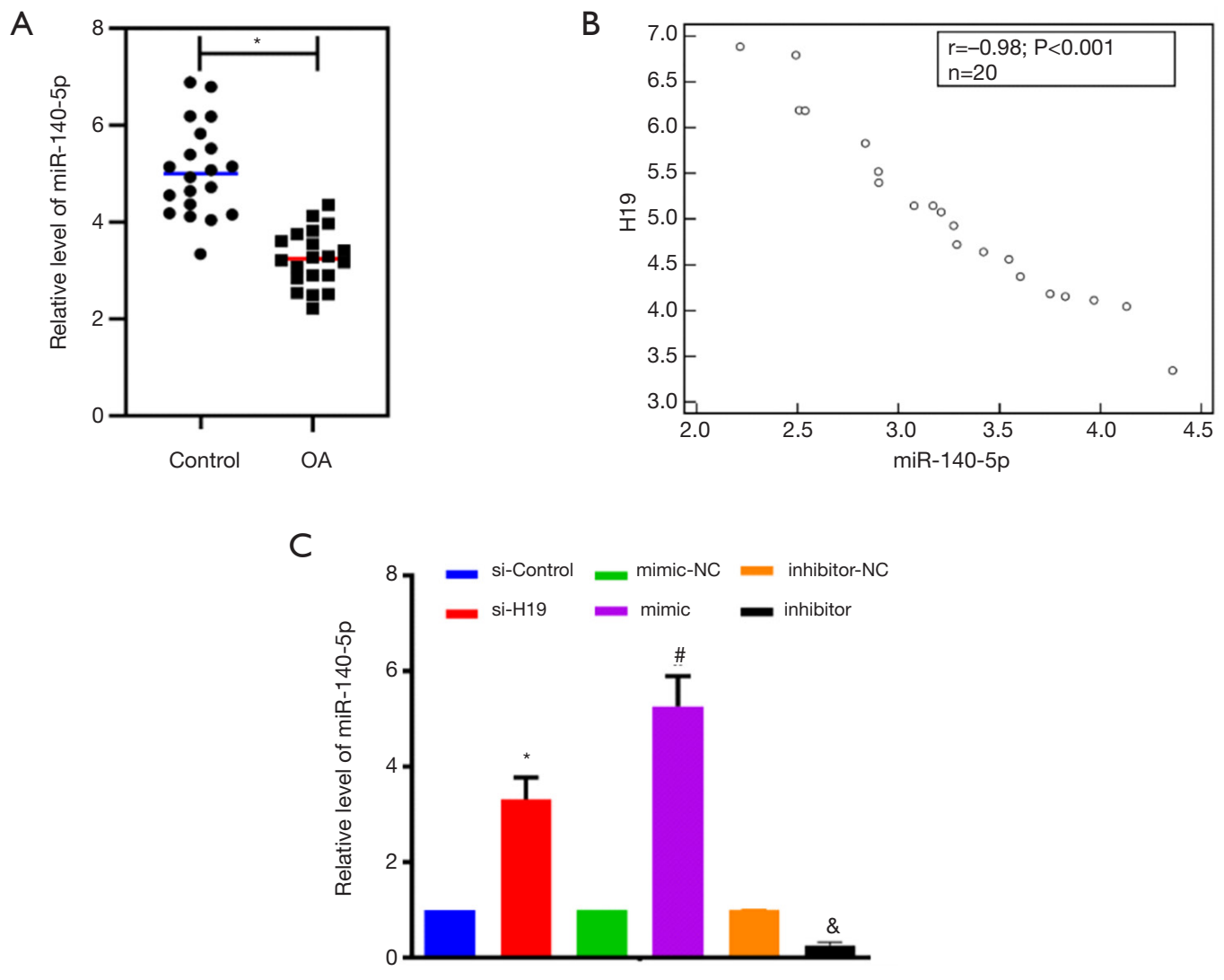

Figure 4 The expression level of miR-140-5p was detected to be negatively correlated with H19 by RT-qPCR. (A) miR-140-5p levels in healthy cartilage tissues and OA cartilage tissues, *, P<0.05. (B) Correlation between miR-140-5p and H19 expression levels in cartilage tissue, $\mathrm{r}=-0.98, \mathrm{P}<0.001$. (C) MiR-140-5p expression levels in chondrocytes with different transfection, compared with si-Control, *, $\mathrm{P}<0.05$; compared with mimic-NC, ${ }^{\#}, \mathrm{P}<0.05$; or compared with inhibitor-NC, ${ }^{\&}, \mathrm{P}<0.05$. OA, osteoarthritis.

Compared with the si-H19 + inhibitor-NC group, mRNA levels of ALP, OCN, and BSP in the miR-140-5p inhibitor + si-H19 group were up-regulated significantly (all $\mathrm{P}<0.05$, Figure $6 A$ ), while the ALP activity of chondrocytes was inhibited, with the difference statistically significant (all $\mathrm{P}<0.05$, Figure $6 B)$.

\section{Discussion}

In this study, the results indicated that high expression of H19 might participate in the development of OA by regulating the process of chondrocyte proliferation, apoptosis, matrix degradation and calcification, which was also closely related to low expression of miR-140-5p. In addition, negative regulation of miR-140-5p was shown to be one of the essential mechanisms by which $\mathrm{H} 19$ was able to regulate degradation and calcification of the OA chondrocyte matrix.

$\mathrm{OA}$, a common joint degenerative disease in the elderly, is characterized by the degradation of cartilage structure and function, with most of the lesions being located in the tissue around the joints, which may eventually lead to joint pain and limited functional activity (8). However, the treatment results and prognosis of $\mathrm{OA}$ are not satisfactory. For example, the most used non-steroidal anti-inflammatory and analgesics may only improve the quality of life by alleviating pain and inflammation. And the intra-articular injection of hormones or hyaluronic acid is only suitable for relieving OA inflammation symptoms (9-11). Therefore, indepth researches about the pathogenesis of OA may supply a detailed theoretical basis for the treatment of this disease.

The MMP family plays a vital role in the pathological process of OA. It is reported that the family of MMP proteases is widely distributed in the human body, with 


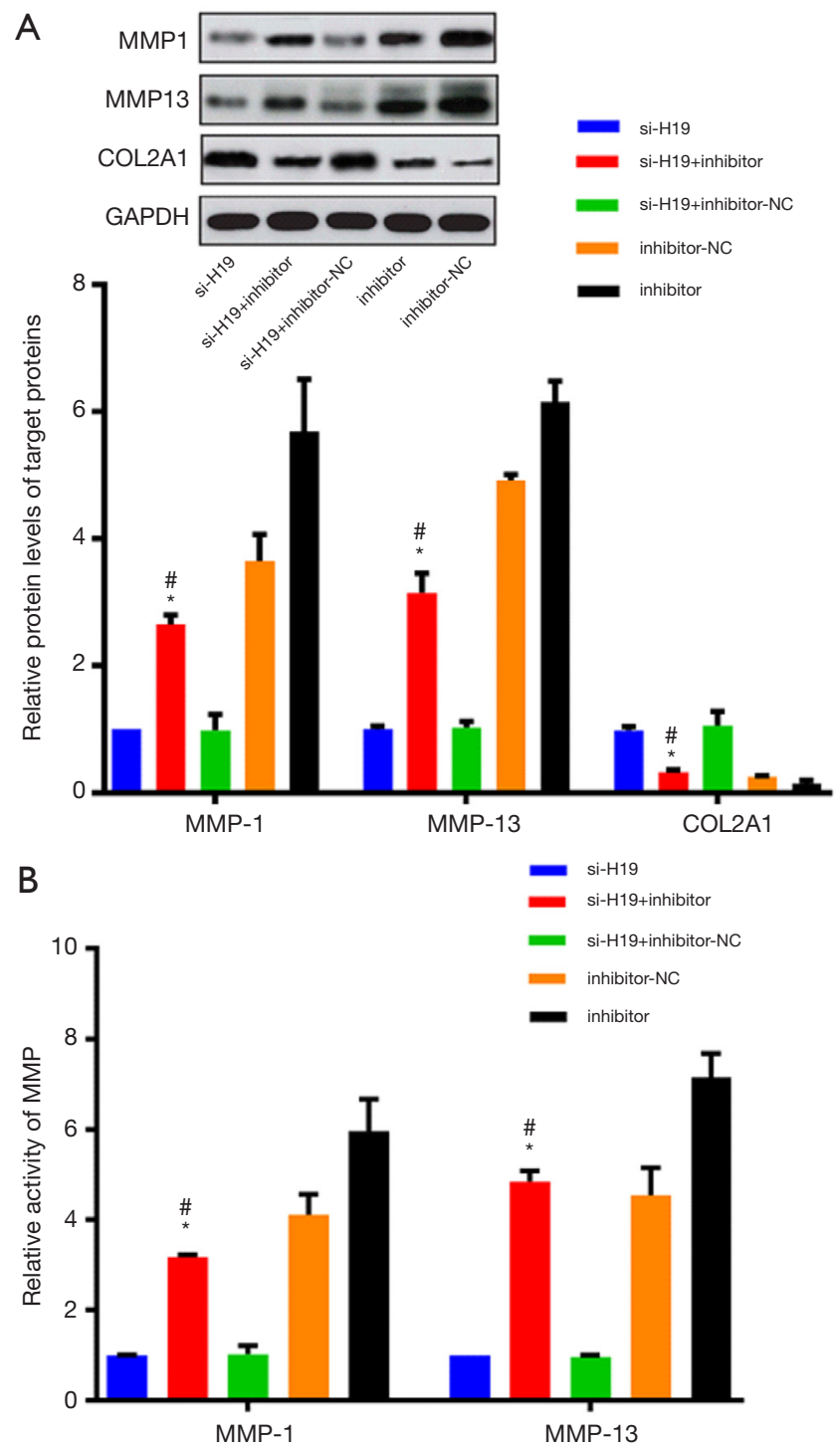

Figure 5 Effect of chondrocytes co-transfected with inhibitor of si-H19 and miR-140-5p on the activity of chondrocyte matrix degradation related indexes MMP-1, MMP-13, COL2A1 and MMP-1 and MMP-13 (A) Western blot detection of MMP-1, MMP-13, COL2A1 protein expression; (B) Kit method to detect the activity changes of MMP-1 and MMP-13. Compared with si$\mathrm{H} 19$, *, $\mathrm{P}<0.05$, compared with si-H19 + inhibitor-NC, ${ }^{\#}, \mathrm{P}<0.05$.

the primary function of controlling the degradation of extracellular matrix components, considered as one of the primary factors in the physiological reconstruction and pathological damage processes $(12,13)$. In OA, MMP is expressed in chondrocytes, synovial cells, and osteoclasts, which is intimately involved in the hydrolysis of cartilage matrix macromolecules, including type II collagen and polysaccharides, and ultimately destroys the structural and functional integrity of the extracellular matrix of articular cartilage. As a kind of collagenase, MMP-1 is one of the rate-limiting enzymes and the most typical type II collagen in the degradation of cartilage matrix, which can degrade collagen in the triple helix region of collagen fibers and induce the activation of other MMP family members to play an essential role in the development of OA (14). MMP13, also known as collagenase 3 , is a newly discovered member of the MMP family and expressed in connective tissue. In healthy cartilage tissues, there is little MMP13 expressed or no expression. Only during the process of cartilage osteogenesis, MMP-13 secretion can degrade the cartilage matrix for osteogenesis. At the same time, it is reported that MMP-13 is a potent protease, which has a particular degradation effect on collagen and non-collagen substances in the extracellular matrix, especially on the type II collagen (15). Therefore, MMP-13 is the most effective protease for cleavage of U-shaped collagen. Recent studies have confirmed that MMP-13 is not only overexpressed in damaged cartilage but also upregulated in synovial tissue, calcified cartilage, and subchondral bone (16). In this study, the expression of MMP-1 and MMP-13 in chondrocytes was down-regulated by overexpression of miR-140-5p or silencing H19, suggesting that H19 might be involved in the development of OA by regulating the extracellular matrix.

Also, in order to confirm the effect of H19 and miR$140-5 \mathrm{p}$ on the calcification of chondrocytes furtherly, we detected the expression levels of ALP, OCN, BSP and the activity of ALP in chondrocytes, finding that expression levels of OCN and BSP were inhibited by H19 silence significantly, as well as the inhibition of ALP activity. Several studies have confirmed that H19 can promote osteogenic differentiation of stem cells and upregulate the expression of OCN and ALP in bone marrow mesenchymal stem cells (17), suggesting that inhibition of H19 expression may inhibit ossification of chondrocytes. Our further research showed that inhibition of miR-140-5p could partially reverse the downregulation of ALP activity, OCN, and BSP expression levels caused by H19 silence. And the promotion effect on osteogenic differentiation of mesenchymal stem cells by inhibition of miR-140-5p was confirmed in the published data $(18,19)$. The result indicated that H19 negatively regulates miR-140-5p in chondrocytes so that miR-140-5p silence could rescue the decreased levels of osteogenic markers (ALP, OCN, and 

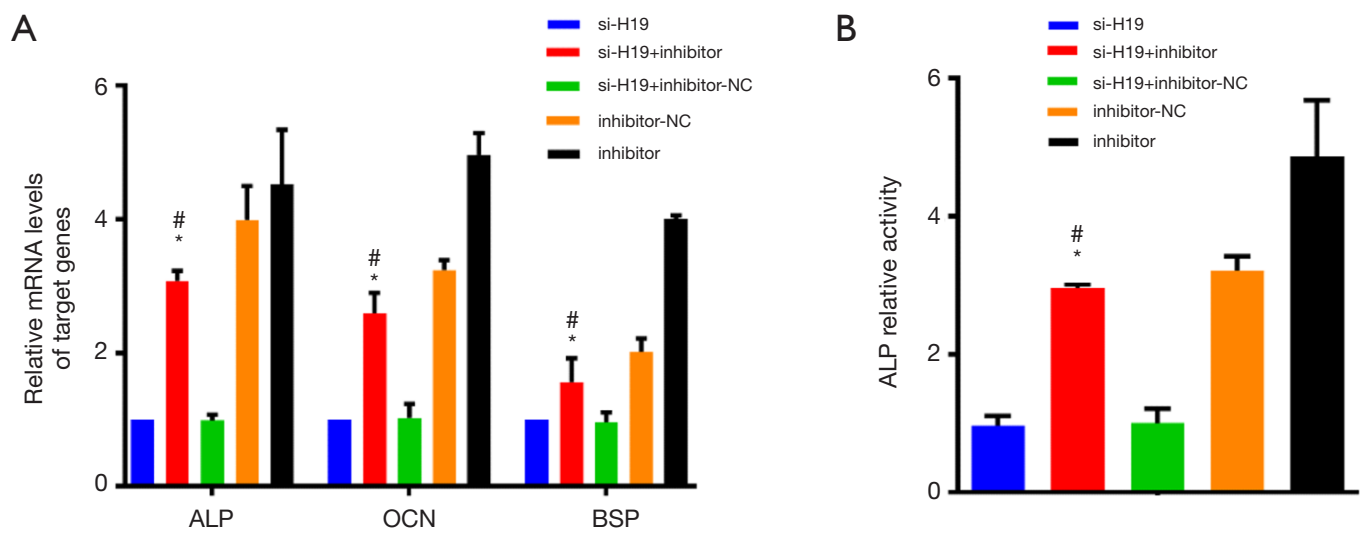

Figure 6 Effect of combined transfection of si-H19 and miR-140-5p inhibitor in chondrocytes on ossification of chondrocytes. (A) The mRNA levels of ALP, OCN, and BSP in chondrocytes were detected by RT-qPCR. (B) The activity of ALP in chondrocytes was detected following the kit method. Compared with si-H19, *, $\mathrm{P}<0.05$; and compared with si-H19 + inhibitor-NC, , $\mathrm{P}<0.05$. ALP, alkaline phosphatase; OCN, osteocalcin; BSP, bone sialoprotein.

BSP) caused by H19 inhibition.

In summary, the results showed that the expression of H19 in OA cartilage tissues was up-regulated, which may promote the degradation and ossification of the extracellular chondrocyte matrix by inhibiting miR-140-5p, thus participating in OA progression.

\section{Acknowledgments}

Funding: None.

\section{Footnote}

Data Sharing Statement: Available at http://dx.doi. org/10.21037/apm-20-929

Conflicts of Interest: All authors have completed the ICMJE uniform disclosure form (available at http://dx.doi. org/10.21037/apm-20-929). The authors have no conflicts of interest to declare.

Ethical Statement: The authors are accountable for all aspects of the work in ensuring that questions related to the accuracy or integrity of any part of the work are appropriately investigated and resolved. The trial was conducted in accordance with the Declaration of Helsinki (as revised in 2013). All specimens were approved by our ethics committee and get the informed consent of the patients.

Open Access Statement: This is an Open Access article distributed in accordance with the Creative Commons Attribution-NonCommercial-NoDerivs 4.0 International License (CC BY-NC-ND 4.0), which permits the noncommercial replication and distribution of the article with the strict proviso that no changes or edits are made and the original work is properly cited (including links to both the formal publication through the relevant DOI and the license). See: https://creativecommons.org/licenses/by-nc-nd/4.0/.

\section{References}

1. DeRogatis M, Anis HK, Sodhi N, et al. Non-operative treatment options for knee osteoarthritis. Ann Transl Med 2019;7:S245.

2. Abbasifard M, Kamiab Z, Sadeghi I, et al. The role and function of long non-coding RNAs in osteoarthritis. Exp Mol Pathol 2020:104407.

3. Kourtis A, Adamopoulos PG, Papalois A, et al. Quantitative analysis and study of the mRNA expression levels of apoptotic genes BCL2, BAX and BCL2L12 in the articular cartilage of an animal model of osteoarthritis. Ann Transl Med 2018;6:243.

4. Mao G, Kang Y, Lin R, et al. Long Non-coding RNA HOTTIP Promotes CCL3 Expression and Induces Cartilage Degradation by Sponging miR-455-3p. Front Cell Dev Biol 2019;7:161.

5. Xu J, Xu Y. The lncRNA MEG3 downregulation leads to osteoarthritis progression via miR-16/SMAD7 axis. Cell Bioscience 2017;7:69.

6. Tao SC, Yuan T, Zhang YL, et al. Exosomes derived from 
miR-140-5p-overexpressing human synovial mesenchymal stem cells enhance cartilage tissue regeneration and prevent osteoarthritis of the knee in a rat model. Theranostics 2017;7:180.

7. Steck E, Boeuf S, Gabler J, et al. Regulation of H19 and its encoded microRNA-675 in osteoarthritis and under anabolic and catabolic in vitro conditions. J Mol Med 2012;90:1185-95.

8. Schmelz M, Mantyh P, Malfait AM, et al. Nerve growth factor antibody for the treatment of osteoarthritis pain and chronic low-back pain: mechanism of action in the context of efficacy and safety. Pain 2019;160:2210.

9. Huang R, Li X, Xu S, et al. Acupoint injection treatment for primary osteoporosis: a systematic review and metaanalysis of randomized controlled trials. Ann Palliat Med 2019;8:586-95.

10. Huang C, Wu Z, Jiang Q. Effect of Qufeng Zhitong capsule on score of pain and knee function in patients with knee osteoarthritis. Chinese Journal of New Drugs 2019;(23):11.

11. Huang K, Wu Z, Zhang Z, et al. Hyaluronic acid,p latelet rich plasma and the combination of both in the treatment of osteoarthritis of the knee. Chinese Journal of Osteoporosis 2019;25:1707-11.

12. Yu Y, Wang C, Liu J, et al. Evolvement of reconstruction rules about extracellular matrix in fibrogenesis after myocardial infarction. Chinese Heart
Journal 2019;31:83-8.

13. Wang H, Ding X, Yang L, et al. Differences in the expression of related degrading enzymes in joint fluids of different stages of osteoarthritis. Journal of Clinical Rehabilitative Tissue Engineering Research 2019;23:3609.

14. Alamgeer, Hasan UH, Uttra AM, et al. Phytochemicals targeting matrix metalloproteinases regulating tissue degradation in inflammation and rheumatoid arthritis. Phytomedicine 2020:153134.

15. Fan K, Jing WU, Qin LI, et al. Advances in matrix metalloproteinase 13 in cartilage remodeling and arthritis. Chinese Pharmacological Bulletin 2018;34:607-11.

16. Dai Y, Lu J, Li F, et al. Changes in cartilage and subchondral bone in a growing rabbit experimental model of developmental trochlear dysplasia of the knee. Connective Tissue Research 2019:1-14.

17. Zhou P, Li Y, Di R, et al. H19 and Foxc2 synergistically promotes osteogenic differentiation of BMSCs via Wnt- $\beta$ catenin pathway. J Cell Physiol 2019;234:13799-806.

18. Hwang S, Park SK, Lee HY, et al. miR-140-5p suppresses BMP2-mediated osteogenesis in undifferentiated human mesenchymal stem cells. FEBS Lett 2014;588:2957-63.

19. Li Z, Jin C, Chen S, et al. Long non-coding RNA MEG3 inhibits adipogenesis and promotes osteogenesis of human adipose-derived mesenchymal stem cells via miR-140-5p. Mol Cell Biochem 2017;433:51-60.
Cite this article as: Yang B, Xu L, Wang S. Regulation of lncRNA-H19/miR-140-5p in cartilage matrix degradation and calcification in osteoarthritis. Ann Palliat Med 2020;9(4):18961904. doi: 10.21037/apm-20-929 